\title{
AN APPROACH TO REMOVE HEAVY METAL IONS IN CERTAIN AQUEOUS SOLUTIONS AND WASTE WATER BY MEANS OF SUITABLE MATRICES AND CHELATORS
}

\author{
Göran Lindgren \\ Inovata $A B$, Sweden
}

\begin{abstract}
The increasing amount of heavy metal ions in nature might not yet be a serious problem. However some of the heavy metals i.e. cadmium, mercury, arsenate (semi-metal) and high concentrations of copper and zinc are clearly toxic to the human body.

The present investigation deals with removal of heavy metal ions in aqueous solution. Different carriers designed for trace analysis as well as for high flow rates will be discussed.
\end{abstract}

\section{KEYWORDS}

Heavy metal ions; Chelating; Trace analysis; Large-scale removal; IMAC

\section{INTRODUCTION}

The idea behind using chelators bounded to a matrix to capture heavy metal ions may be related to a publication from 1975 by J. Porath. In this investigation [1] different chelators immobilised to an agarose matrix and different heavy metal ions were used. It was shown that the heavy metal ions interacted with histidine and cysteine amino acids of a protein. Furthermore by using chelators with several binding sites the protein could be more or less adsorbed to the matrix. The technique was named IMAC (Immobilised Metal Affinity ion Chromatography) and it was shown that histidine containing proteins could be purified in one single step which reduced both time and costs. Agarose is a polysaccharide refined from the seaweed agar agar and used as a hydrophilic polymer in separation and purification of biomolecules

Mid 1990 Inovata together with J. Porath developed a series of separation media based on rigid agarose [2] with different chelators [3]. Iminodiacetic acid (IDA), Figure 1, is the most common chelator.

Figure 1. Iminodiacetic acid IDA.

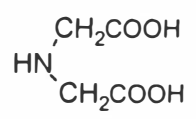




\section{Kalmar ECO-TECH '05 and \\ The Second Baltic Symposium on Environmental Chemistry \\ KALMAR, SWEDEN, November 28-30, 2005}

The metal ion will interact with the electron pair of the nitrogen atom and with the two carboxyl groups thus leaving three orbitals for interaction with a protein. IDA is a trivalent chelator. Another useful chelating group is shown in Figure 2.

$$
\mathrm{NH}_{2} \mathrm{CH}_{2} \mathrm{CH}_{2}-\mathrm{N}^{\prime} \mathrm{CH}_{2} \mathrm{CH}_{2} \mathrm{NH}_{2}
$$

Figure 2. Tris(aminoethyl)amine (TREN)

In TREN the metal ion is strongly bound to the chelator, cf. EDTA, and the protein can easily be desorbed from the matrix during mild condition.

To get a high capacity for metal ions polyethyleneimine (PEI) can be used. PEI contains repeating $-\mathrm{NH}\left(\mathrm{CH}_{2} \mathrm{CH}_{2}\right) \mathrm{NH}_{2}$ groups and is similar to TREN with strong interaction. PEI can be made even stronger by reaction with bromoacetic acid.

Interaction between a metal ion and a polymer substituted with TREN may be represented as in Figure 3.

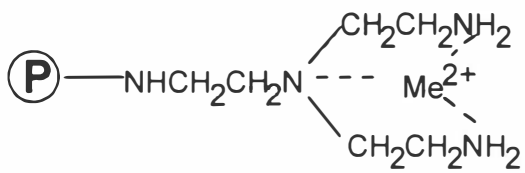

Figure 3. Electrostatic interaction between a metal ion and a chelating polymer

The electrostatic forces between a metal ion and a chelator are much stronger compared to ordinary ion exchange. In certain water the ion strength is high due to presence of salt and the metal ion will be eluted from a non-chelating matrix. This might also occur if the flow right is to high. However it can be shown that using a chelating group rather high concentration of salt can be used. Desorption occurs when the $\mathrm{pH}$ of the mobile phase is decreased below 2 .

\section{MATERIALS AND INSTRUMENTATION}

For small scale experiments commercially available agarose gels from Inovata $A B$ in Sweden was used (IDA-Novarose, TREN-Novarose, PEI-Novarose). The liquid chromatography system (pump, injector and detector) was from LKB Produkter AB in Sweden.Determination of copper was made with an ion selective copper electrode from Thermo Electron in USA. ICP-AES and FAAS was performed at Uppsala University, Tekniska verken, Linköping and by Alcontrol in Linköping. Bark from pinus silvestris was processed by Tekniska verken, Linköping. 


\section{RESULT AND DISCUSSION}

Heavy metal ions can be found in many different kinds of water. Depending on the concentration of metal ions and type of water and on the purpose of removal, the choice of chelating matrix is of importance.

An ordinary liquid chromatography system consisting of glass columns, injector, pump and detector were used for checking capacity and adsorption of heavy metal ions throughout the laboratory experiments. Copper(II)sulphate dissolved in water at about 50 mmolar concentration was injected in $100 \mu \mathrm{l}$ portions to determine the capacity of the matrix. The concentration of copper was determined with an ion selective copper electrode.

Our first approach was to investigate the existing chelating agarose produced for protein separation.

Polyethyleneimine covalently bound to agarose was chosen as a model. The matrix consists of a spherical particle of $40 \mu \mathrm{m}$ size and packed in small columns $(5.7 \times 15 \mathrm{~mm})$. The intention was to investigate the possibility to ultratrace analysis of tap water or other types of water with very low concentration of heavy metal ions. The highest capacity was obtain with PEI agarose $(500 \mu \mathrm{ekv} / \mathrm{ml})$ simply determined by injection of copper(II)sulphate of known concentration into a column and measure the blue band with a ruler. A $1 \mathrm{ml}$ column can thus adsorb heavy metal ions from hundreds of litres of water containing heavy metal ions on a ppb level.

\subsection{Trace analysis}

A deep investigation using small columns packed with commercially available chelating matrix was made by Hashemi et.al [4] at the university of Uppsala.

He studied capacities, recovery and kinetic for several chelating matrix with different metal ions. To check the reliability of their experiments a field experiment were performed. They constructed a simple column by placing two frits at the outlet of a $50 \mathrm{ml}$ syringe. Between the two frits a small sample of PEI agarose matrix were placed. Figure 4.

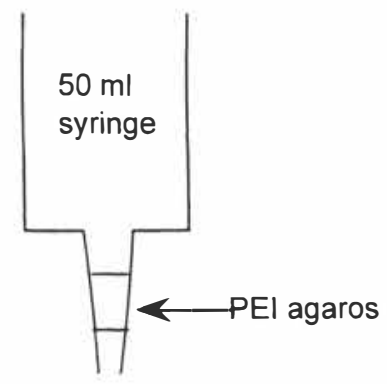

Figure 4. Syringe with PEI agarose for pre-concentration of tap water 
Kalmar ECO-TECH '05 and

The Second Baltic Symposium on Environmental Chemistry

KALMAR, SWEDEN, November 28-30, 2005

Table 2. Copper concentrations in tap waters from the Uppsala region

\begin{tabular}{|c|c|c|c|}
\hline \multirow[b]{2}{*}{$\begin{array}{l}\text { Sample } \\
\text { No }\end{array}$} & \multicolumn{3}{|c|}{ Copper concentrations $(\mu \mathrm{g} / \mathrm{l})$} \\
\hline & ICP-AES & FAAS & $\begin{array}{l}\text { Recovery } \\
\% \text { from the column }\end{array}$ \\
\hline 1 & 118 & 113 & 95.8 \\
\hline 2 & 45.8 & 47.8 & 104 \\
\hline 3 & 17.4 & 14.6 & 84.1 \\
\hline 7 & 655 & 640 & 97.7 \\
\hline 8 & 311 & 285 & 91.6 \\
\hline 9 & 194 & 176 & 91.2 \\
\hline 10 & 403 & & \\
\hline 20 & 176 & 202 & 115 \\
\hline 21 & 237 & 227 & 95.9 \\
\hline 22 & 8.2 & 8.1 & 99.0 \\
\hline
\end{tabular}

The syringe was filled with tap water containing a known concentration of copper and pressed out through the frits. The column was eluted with $1 \mathrm{M} \mathrm{HCl}$ and analysed with flame atomic absorption spectrometry (FAAS). Several test experiments with different concentration of copper was done and analysed. The recovery was shown to be as good as $99,3 \%$

An investigation was made on 22 households in Uppsala. Tap water was allowed to flash for 10 minutes where after the syringe was filled to the $50 \mathrm{ml}$ mark and passed slowly through the column by means of the plunger. The background for this investigation was that high $\mathrm{Cu}$ concentration in tap water might be harmful for small children if used for cooking.

Table 1. shows some of the results from the investigation. All samples was analysed both directly with inductively coupled plasma (ICP-AES) and with FAAS after elution of the column with $\mathrm{HCl}$. Tap water in Uppsala is known to dissolve copper from the distribution pipes.

Sample 22 was taken from a tap where no copper tubing was installed. The highest value was as high as $655 \mathrm{ppb}$. Tap water with such a high values is not suitable for preparation of food for small children (might cause diarrhoea).

\subsection{Medium scale}

The fast kinetics shown during trace analysis encourages us to look at larger particle that can withstand higher flow rates and larger volumes of contaminated water. Spherical and cross-linked agarose is very expensive and cannot be used in larger scale. Since agarose is produced from agar 
Kalmar ECO-TECH '05 and

The Second Baltic Symposium on Environmental Chemistry

KALMAR, SWEDEN, November 28-30, 2005

Table 2. Result from 8 litres of water from a dental practice.

\begin{tabular}{llll}
\hline Metal ion & Incoming water & Outgoing water & $\begin{array}{l}\text { Adsorbed } \\
\text { on column }\end{array}$ \\
\hline $\mathrm{Hg}$ & $264 \mu \mathrm{g}$ & $<0.8 \mu \mathrm{g}$ & $>263.2 \mu \mathrm{g}$ \\
$\mathrm{Ag}$ & $48 \mu \mathrm{g}$ & $<8 \mu \mathrm{g}$ & $>40 \mu \mathrm{g}$ \\
$\mathrm{Sn}$ & $624 \mu \mathrm{g}$ & $40 \mu \mathrm{g}$ & $584 \mu \mathrm{g}$ \\
$\mathrm{Zn}$ & $4560 \mu \mathrm{g}$ & $112 \mu \mathrm{g}$ & $4448 \mu \mathrm{g}$
\end{tabular}

we decided to make agar particles in the same way as for agarose. A larger rigid particle will give lower backpressure and allows higher flow rates.

A $200 \mu \mathrm{m}$ rigid agar particle will give a reasonable high flow rate in moderate sized columns. The agar particle was reacted with PEI in the same way as for agarose [5].

Looking for suitable application we found that the dentist practices use a mechanical separation of amalgam. Amalgam consists of mercury, silver, zinc and tin. During transport in the water pipes from the dental pratice some of the metal will come out from the mechanical separation in ionic form with the wash water. The excess of water goes directly down the drain.

8 litres of water from the mechanical separator was collected and allowed to pass a $25 \times 100 \mathrm{~mm}$ glass column. Samples was taken before and after the column and analysed with ICP-AES. Elution of the column with acetic acid released all the metal ions and the fraction was analysed with the same method, Table 2.

Assuming that the water was collected from an average dental practise a calculation of the total amount of mercury coming out into the waste water at the water purification plant is about $5 \mathrm{~kg}$ a year.

The total capacity of a one-litre PEI-Agar column is enough to trap the metal ions from amalgam during one year in a normal dental practise. Such a system were constructed and connected to the outlet of an amalgam separator.

Other examples. Incoming water to a glassworks showed too high values of aluminium. Samples were taken and a test with the PEI-Agar gave total adsorption.

To be able to make field tests a pilot scale mobile apparatus consisting of two 10 litres columns filled with PEI-Agar was then constructed. The instrument has a complete set up with pumps and on-line $\mathrm{pH}$ measurement. The idea is to connect the system to the outgoing water system by a split from a factory heaving high levels of heavy metal ions. When the first column is saturated the second column is automatically placed in the main stream. The first column is regenerated with acetic acid and $\mathrm{pH}$ adjusted. Knowing the metal ion content of the incoming water and the uptake of the columns the size of a filter system fitting the need can be estimated. 


\author{
Kalmar ECO-TECH ${ }^{\prime} 05$ and \\ The Second Baltic Symposium on Environmental Chemistry \\ KALMAR, SWEDEN, November 28-30, 2005
}

\title{
3.4 Large scale
}

A draw back of a column containing $200 \mu \mathrm{m}$ particles is that the incoming water must be relatively free from particles. A pre filter must be used but even than problems with clogging of the column might occur.

Tekniska Verken in Linköping has developed a method of removing oil in contaminated water. They used bark from pinus silvestris. The bark was made hydrophobic by coating with a hydrophobic compound. The hydrophobic surface forced the water out of the pores of the bark thus giving oil free entrance to be adsorbed.

Before coating the bark is grinded and classified to a certain size. The size of the bark particles determines the flow rate that can be used in a process.

Laboratory experiment on bark treated in a special way using the same PEI coating technique as for agar gave a product with enough capacity to be used in large scale. The surface of bark (inner and outer surface) contains cellulose, lignin and some substituted aromatic compounds. By treatment with sodium hydroxide some of the cellulose will hydrolyse and form carboxyl groups. PEI is than adsorbed by ion exchange and stabilised [6].

In a large scale experiment several cubic meters of bark coated with PEI was produced. The bark was placed in four filters each filter containing 700 litres. In certain thermal power stations the smoke from the furnace is treated with water to trap the impurities. If the wood contains metal some of the metal will ionise and be trapped in the condensate water. The filter system was constructed to fit the volumes and flow rates coming out from a thermal power station. In this special case the outlet water from the power station the amount of zinc was above the permitted level. During a two-month period 2200 cubic meter of water flow through the filter and $8.8 \mathrm{~kg}$ of zinc was trapped [7].

\section{CONCLUSION}

It has been shown that chelating groups like IDA, TREN or PEI covalently bound to a suitable matrix can purify water from heavy metal ions at different volumes and flow rates in an efficient way. Trace analysis by using a high capacity chelating matrix can simplify collection of ions in large amount of water for FAAS or ICP-AES analysis.

Removal of heavy metal ions from contaminated water may be of great importance in the future. Simple chemical treatment of bark or similar inexpensive carriers gives a low cost product which is possible do use at high flow rates and with reasonable capacity.

\section{REFERENCES}

[1] Porath, J., 1975. Nature 258, 598-599.

[2] Inovata AB, 1986. US Patent No. 4,973,683

[3] Inovata AB, 1992. Product Data Sheet, Novarose IMAC-gels and columns.

[4] Hashemi, P., 1997. Agarose Based Chelating Adsorbents for Preconcentration of Trace and Ultratrace Heavy Metals in Water. Acta Unuversitatis Upsaliensis 1-4d.

[5] Inovata AB, 1994. EP-application No. 94908124.4

[6] Inovata AB, 2005. Patent applied for.

[7] Andersson, A. 2004. Tekniska verken, Linköping. Personal communication. 